\title{
INTERACTION OF CORONAL MATERIAL WITH MAGNETIC FIELDS
}

\author{
G. W. PNEUMAN and ROGER A. KOPP \\ High Altitude Observatory, National Center for Atmospheric Research* Boulder, Colo., U.S.A.
}

\begin{abstract}
The optical resolution in recent eclipse photographs is now sufficient to illustrate clearly many qualitative features of gas-magnetic field interactions in the solar corona. Close to the Sun where the field is strong, the coronal gas over certain regions of the solar surface can be contained within closed loop structures. However, since the field strength in these regions declines outward rapidly, the pressure and inertial forces of the solar wind eventually dominate and distend the field outward into interplanetary space. The overall configuration is determined by a complex interplay of inertial, pressure, gravitational, and magnetic forces. The present work is oriented toward the understanding of this interaction.

Of central importance to this general problem is the typical 'helmet' streamer generally overlying a large diffuse bipolar region on the solar surface. A helmet streamer consists of closed magnetic loops near the Sun and open field lines adjacent to and above the loops. The configuration possesses a 'cusp-type' neutral point (to be differentiated from a $y$-type neutral point) at the top of the closed loops. In addition to volume currents, a single sheet current separates the regions of opposite polarity above the neutral point. Below the neutral point, sheet currents also are present between the open and closed regions. These sheet currents are necessary to support the discontinuity in gas pressure which arises since the open region is expanding and the closed region is not. The neutral point problem is discussed in some detail and sample numerical examples are presented.
\end{abstract}

The interaction of coronal material with the magnetic fields of the Sun is quite evident in recent white light eclipse photographs of the inner corona. For example, large, closed magnetic loops are visible over extensive regions which presumably completely inhibit coronal expansion. Beyond about $2 R_{\odot}$, however, the field lines are all open, allowing streaming out into interplanetary space.

This observed picture is consistent with two theoretical concepts. Firstly, due to the high electrical conductivity and the large scale of coronal structures, the magnetic Reynolds numbers appropriate for these conditions are enormous. Consequently, the magnetic fields are effectively frozen into the material. Secondly, the ratio of magnetic pressure to dynamical pressure evidently varies from a value somewhat greater than one at the coronal base to values much less than one at large distances, with the two being equal somewhere near the tops of the closed loop systems.

In this work, we attempt to describe within the context of a theoretical model this complex interplay of inertial, pressure, gravitational, and magnetic forces. In order to simplify the problem as much as possible without undermining the relevant physics, we will consider the corona as being isothermal and symmetric about the rotation axis. Also, we neglect the effects of solar rotation.**

* The National Center for Atmospheric Research is sponsored by the National Science Foundation.

** Although rotation introduces a toroidal magnetic field in the corona, it can be shown to have a negligible influence on the poloidal components of field and velocity which are of interest here. 


\section{The Helmet Streamer}

Of central importance to the general problem of the interaction of the solar wind with the coronal magnetic fields is the so-called 'helmet' streamer. This structure is of special interest since, by definition, it represents the transition from closed to open field lines and requires special considerations which distinguish it from totally open magnetic configurations. These distinguishing considerations are the neutral point existing at the top of the closed loops and the sheet currents both above the neutral points where the field changes polarity and below the neutral point on the interface between the open and closed regions. This helmet configuration is schematically sketched in Figure 1.

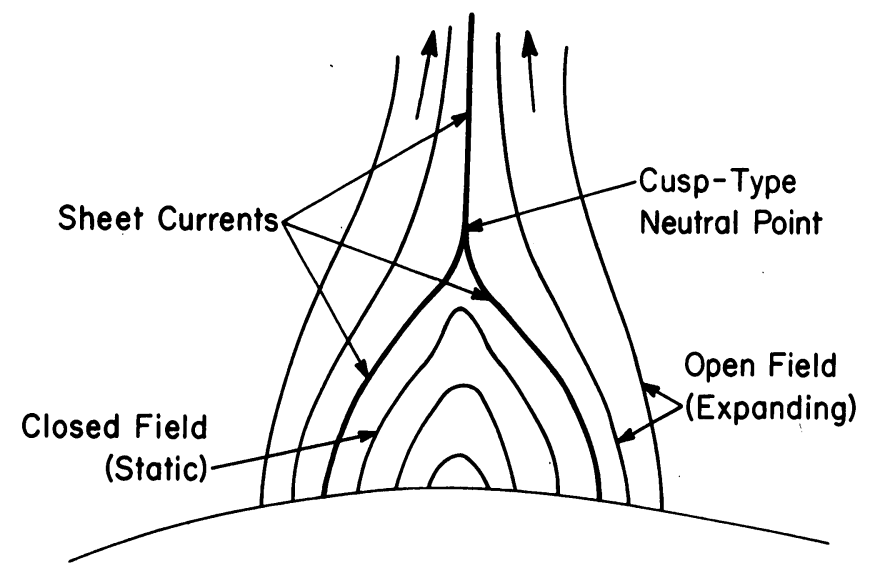

Fig. 1. Schematic diagram of a helmet streamer configuration.

Since motion of material across field lines is essentially prohibited under coronal conditions, we can safely assume that coronal expansion is not taking place in the closed magnetic region (if, indeed, the field lines themselves are stationary). Likewise, conductive losses across the field are insignificant. If radiative losses are negligible, conditions should be almost isothermal there and the gas pressure determined by the equation of hydrostatic equilibrium applied along the field.*

In the region of open field lines, outward heat conduction and expansion are taking place. These processes cause the temperature to decline outward there. This decline results in more rapid pressure drop with height in the open region than in the closed region and is directly responsible for the large observed outward increase of density enhancement in helmet streamers (Pneuman and Kopp, 1970).

Since the gas pressure is falling faster in the open region than in the immediately adjacent closed region, there is a pressure discontinuity at the interface separating the regions. The quantity $p+B^{2} / 8 \pi$, however, must be continuous across the interface. Hence, there must be a jump in $B^{2} / 8 \pi$ equal and opposite to the jump in $p$. In the limit

* By this, we mean that the pressure at any given point in the closed region is related to the base pressure only on the same field line and not, for example, to the pressure directly below. 
of infinite electrical conductivity, a sheet current must exist at the boundary to produce this jump in field strength. There must also be a sheet current at the neutral sheet above the neutral point separating the regions of opposite magnetic polarity.

The field configuration in the vicinity of the neutral point can theoretically be of three types (Sturrock and Smith, 1968), two of which can be ruled out by physical considerations. The ' $y$-type' neutral point can be ruled out since, for this case, the field vanishes when approaching the neutral point from all directions and there is consequently no way to balance the pressure jump between the open and closed regions. In the 'inverted $T$-type' neutral point, the field vanishes in the open region but not in the closed region and the jump in $B$ is of the wrong sign. The only type of neutral point consistent with the forces involved is the 'cusp-type' geometry in which the field goes to zero approaching the neutral point from the inside, but does not vanish on open field lines. The condition to be met there can, therefore, be mathematically stated as

$$
p_{c}=p_{s}+B_{s}^{2} / 8 \pi
$$

where the subscript $c$ refers to the closed region and the subscript $s$ to the streaming region.

Finally, we point out another interesting property of the neutral point. If the sonic point lies well above the cusp (this is probably the case under actual coronal conditions), then it can be shown that the pressure difference between the open and closed regions at the neutral point is about equal to the kinetic energy density in the solar wind just outside the closed region (Pneuman and Kopp, 1971). Since this pressure difference at the cusp must also balance the magnetic pressure just outside (see Equation (1)), we have

or

$$
\frac{1}{2} \varrho_{s} V_{s}^{2} \approx B_{s}^{2} / 8 \pi
$$

$$
V_{s} \approx B_{s} / \sqrt{4 \pi \varrho_{s}}
$$

Hence, for the expansion just outside the closed region the Alfvénic point occurs at the cusp.

\section{Numerical Method of Analysis}

Having discussed the physical aspects of closed and open magnetic structures, we now turn to the general problem of numerically determining the properties of a model corona subject to specified boundary conditions at the coronal base. The necessary base conditions are density, temperature, and normal component of the magnetic field. Also, some appropriate model of coronal heating must be incorporated.

To solve the relevant differential equations, we employ an iterative technique. The iterative loop consists of three distinct steps, each of which is manageable using an electronic computer. A detailed description of the iterative method appears elsewhere (Pneuman and Kopp, 1971). We only briefly describe the contents of the three steps here. 
The first step consists of calculating the magnetic field configuration for a prescribed distribution of volume currents $\mathbf{J}$ and sheet currents $\mathbf{J} *$ over all space as well as the specified normal component of the magnetic field at the solar surface. Since $\nabla \cdot \mathbf{B}=0$, we employ the vector potential $\mathbf{A}$ in which

$$
\nabla \times(\nabla \times \mathbf{A})=4 \pi \mathbf{J}
$$

This differential equation for $\mathbf{A}$ is of the elliptic type and is integrated using standard relaxation methods. For axially symmetric distributions, both $\mathbf{A}$ and $\mathbf{J}$ have only azimuthal components, $A$ and $J$.

In this analysis, we treat only field configurations which are symmetric about the equator. The conditions to be met at the neutral point in this case are that

$$
\frac{\partial}{\partial r}(\operatorname{Arsin} \theta)=\frac{\partial^{2}}{\partial r^{2}}(\operatorname{Arsin} \theta)=0,
$$

where $\theta$ is the colatitude and $r$ the radial distance from the solar center. For a given set of electric currents, these two conditions can be simultaneously satisfied at only one point, thus providing a unique solution.

The magnetic field configuration determined in step 1 defines a streamtube geometry in which the solar wind equations may be integrated outward along each field line. Solution of these equations in this geometry is greatly facilitated by the fact that there are no magnetic forces along the field. On closed flux tubes, hydrostatic equilibrium holds along the field and the velocity is taken to be zero. On open field lines, we find the so-called 'critical' solution in which the solar wind starts subsonically near the Sun, passes smoothly through the critical point, and is supersonic at large distances.

The integration of the equations for conservation of momentum and mass along the field in step 2 determines all the necessary physical variables. However, the equilibrium of forces normal to the field has not yet been imposed. In step 3, we use this condition to determine new electric currents. In other words, we determine an electric current $\mathbf{J}$ which in conjunction with the magnetic field $\mathbf{B}$ determined in step 1 , produces a $\mathbf{J} \times \mathbf{B}$ force necessary to balance the components of the pressure, inertial, and gravitational forces normal to the field.

Having found new expressions for the current density in step 3, the iterative loop is complete and we can return to step 1 continuing the process. The iteration is considered converged when the field on a given iteration differs from that of the previous iteration by some specified small amount.

\section{A Numerical Example}

The field line configuration of a sample converged solution using the iterative technique of Section 2 is shown in Figure 2. Here, the normal component of the field at the surface is that of a dipole of strength $1 \mathrm{G}$ at the pole. The gas pressure at the surface is taken to be independent of latitude and equal to the surface magnetic pressure at the pole. The coronal temperature is chosen to be $1.56 \times 10^{6} \mathrm{~K}$ and also independent of 


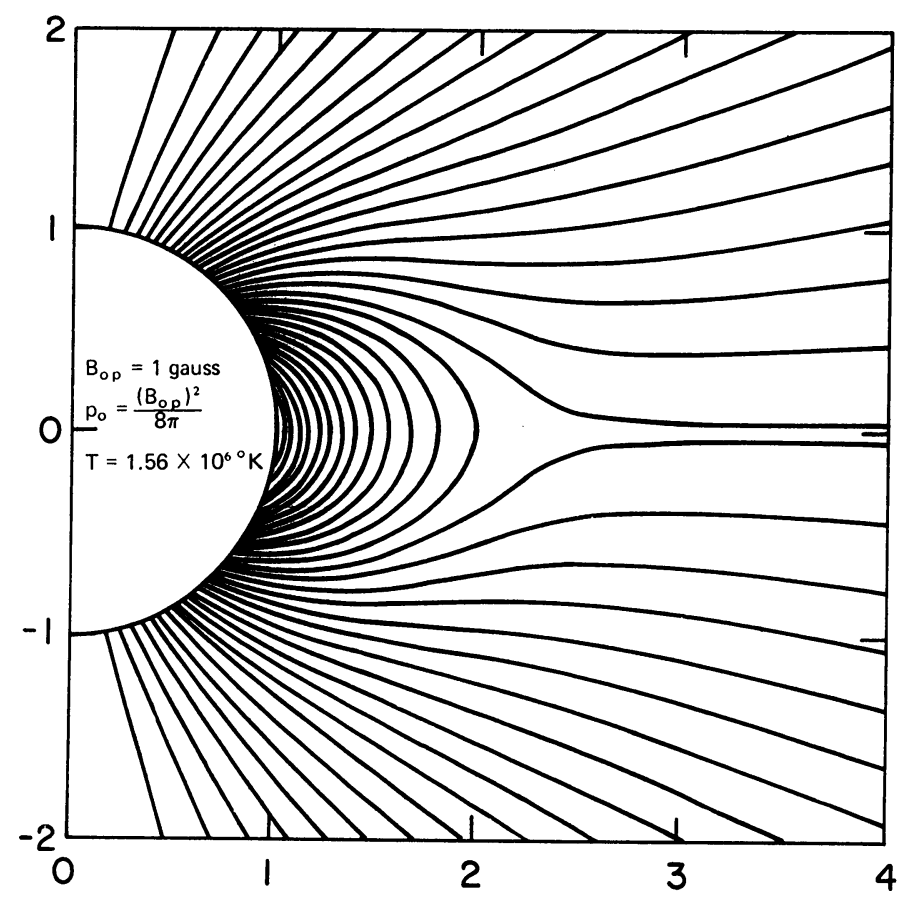

Fig. 2. Field and streamline distribution corresponding to base conditions in which the normal component of the magnetic field is that of a dipole and the reference density is independent of latitude.

latitude. The computed configuration is similar in many respects to the large helmet streamers observing during solar eclipses. Closed loops extend out to about $2.5 R_{\odot}$, the field everywhere being distended outward by the solar wind. The height at which the field lines open at the equator is most sensitive to coronal temperature due to its influence on the scale height.

A more instructive illustration than Figure 2 is Figure 3, in which the same solution is plotted in a different coordinate space. The ordinate here is $\cos \theta$ and the abscissa $R_{\odot} / r$. The advantage of this system is that the solution over all space can be shown in a finite box. The top and bottom boundaries represent the poles, the righthand boundary the solar surface, and the left-hand boundary infinity. We see that the field becomes asymptotically radial at large distances. The nearly vertical dashed curve depicts the sonic surface and the solid curve the Alfvenic surface. The sonic surface is at $4 R_{\odot}$ at the pole and moves out to $5 R_{\odot}$ near the equator. The Alfvénic surface, on the other hand, is at about $5 R_{\odot}$ at the pole and moves in to the cusp at the equator $\left(\approx 2.5 R_{\odot}\right)$, as expected from the discussion in Section 1 .

The variation of solar wind velocity with latitude is shown at two different heights in Figures 4 and 5. Figure 4 depicts the flow at a typical height below the top of the helmet with the region of zero velocity indicating the region of closed field lines. The velocity on open field lines is seen to increase from the pole toward the equator. In Figure 5, representing a level above the neutral point, the variation of velocity with 


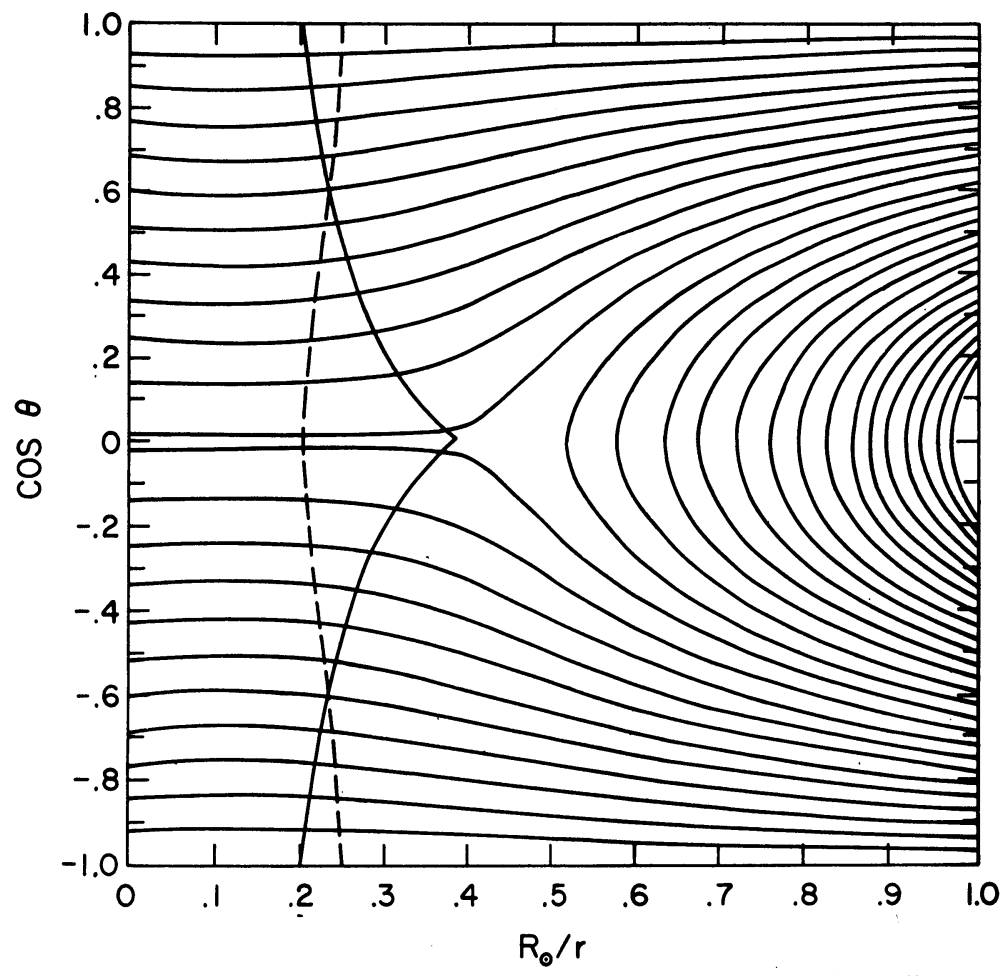

Fig. 3. The same field configuration as in Figure 2 but plotted with $\cos \theta$ as the ordinate and $R_{\odot} / r$ as the abscissa. The vertical dashed curve represents the sonic surface and the solid curve the Alfvénic surface.

latitude is the opposite, with the lowest velocity occurring at the equator. This qualitative difference in velocity distribution is due to the behavior of the flux tube cross section. Below the neutral point, the cross section expands more rapidly near the equator than at the pole. Above the neutral point, the opposite is the case.

\section{Conclusions}

The results of Section 3, in spite of applying only to an extremely simple configuration symmetric both to the rotational axis and the equator, nevertheless demonstrate the possibility of numerically modeling the structure and physical properties of the solar corona and interplanetary medium as a function of measured quantities at the coronal base. The obvious extensions which must be made are the inclusion of the energy equation within the framework of the model as well as relaxing the symmetry conditions imposed here. When this is done, more realistic surface distributions of magnetic field and density can be treated.

\section{Acknowledgements}

The authors wish to offer special thanks to Nancy Werner who did the computer programming for this work. 


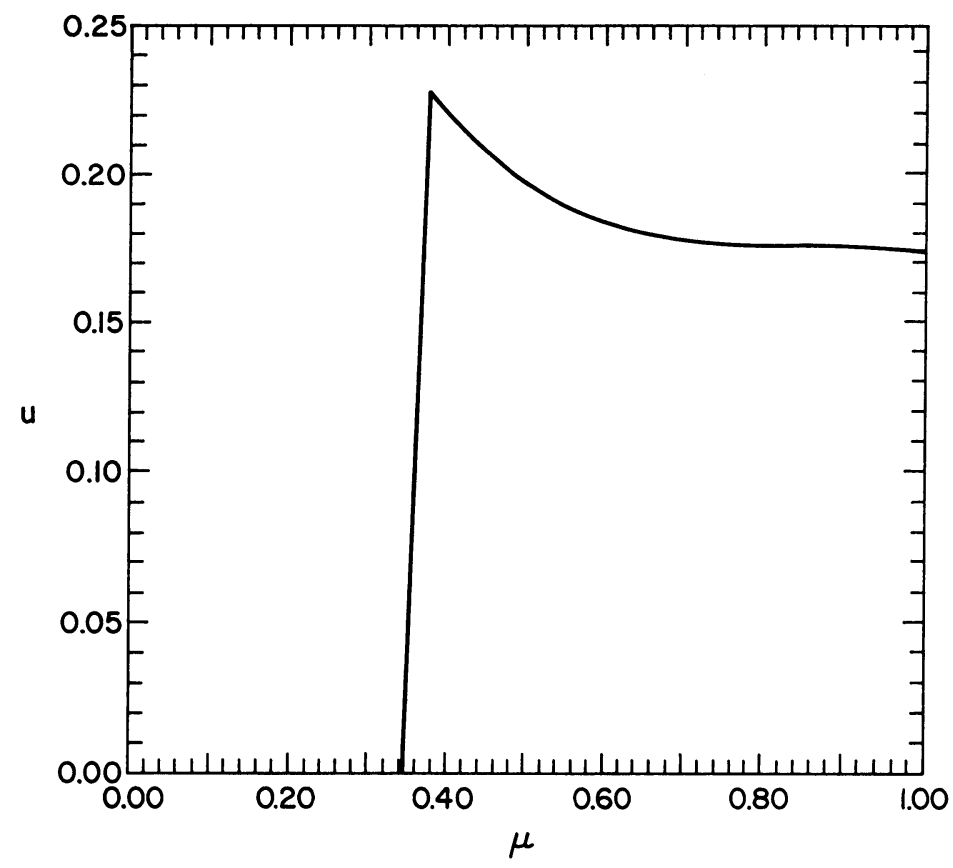

Fig. 4. Variation of velocity with latitude at a typical level below the top of the helmet. Here $u=V / \sqrt{2} V_{s}$, where $V_{s}$ is the sound speed, and $\mu=\cos \theta$. The region of zero velocity is the region of closed field lines.

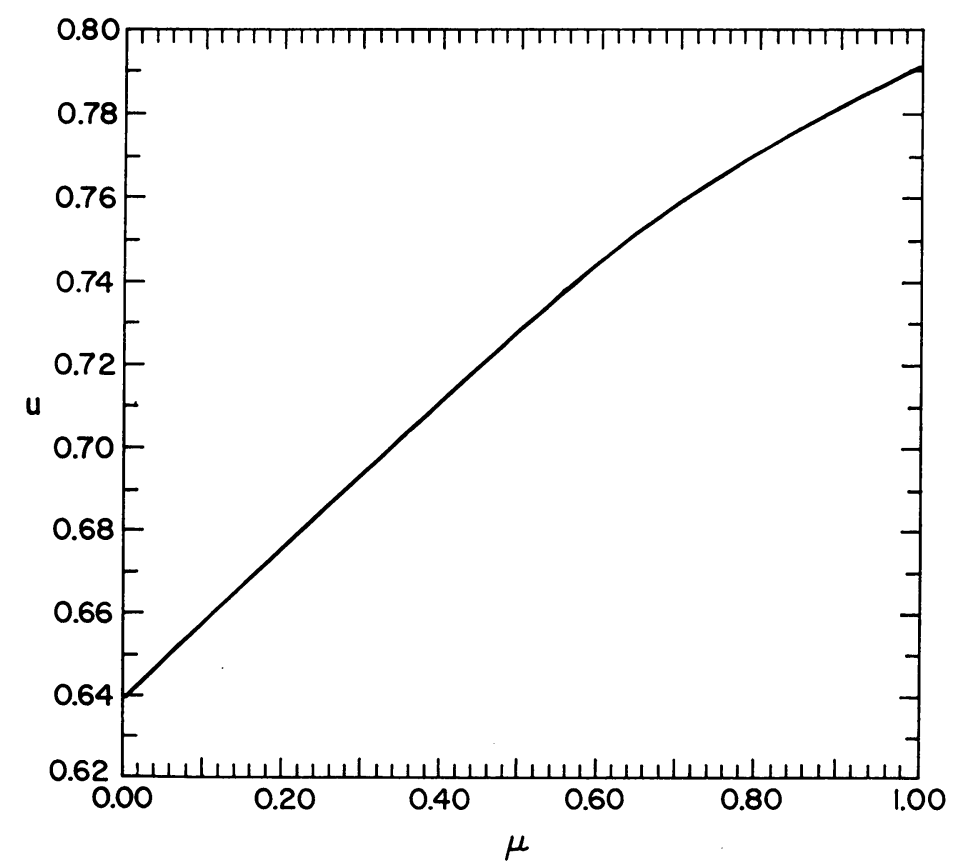

Fig. 5. Variation of velocity with latitude at a typical level above the neutral point. Note that, as opposed to Figure 4, the velocity here decreases from pole to equator. 


\section{References}

Pneuman, G. W. and Kopp, R. A.: 1970, Solar Phys. 13, 176.

Pneuman, G. W. and Kopp, R. A.: 1971, Solar Phys. 18, 258.

Sturrock, P. A. and Smith, S. M.: 1968, Solar Phys. 5, 87.

\section{Discussion}

Kuperus: The neutral sheet type magnetic field configuration which you used in your model has the complication of being unstable due to a magnetohydrodynamical instability called the tearing mode instability. The result of this instability is that field and matter might be dragged towards the neutral sheet where the field is partly annihilated. The first remark is that this effect might considerably change your field topology close to the 'neutral' sheet. My second remark is that due to the enhanced annihilation of the magnetic field in the sheet layer an extra heating term enters your energy equation thereby modifying the expansion of the outer corona.

Pneuman: I don't know whether the neutral sheet in this configuration is stable or unstable. A stability analysis has not been carried out to answer this question. If such an instability exists, it is probably relevant only in the near vicinity of the sheet and will not affect the overall structure which is of interest here. That this must be true is borne out by the observation that these helmet streamers appear very stable for several rotations and seem to evolve only as the underlying fields change their configuration.

In answer to your second remark, the example shown here is for an isothermal corona and energy considerations have not yet been included in the analysis. The point you raise, however, is an interesting one and may indeed be an important consideration in the central regions of streamers. 